\title{
Optical Wave Modes: Localized and Propagation-Invariant Wave Packets in Optically Transparent Dispersive Media
}

\author{
MIGUEL A. PORRAS \\ Universidad Politécnica de Madrid, Madrid, Spain \\ PAOLO DI TRAPANI \\ Università degli studi dell' Insubria, Como, Italy \\ WEI HU \\ South China Normal University, Guangzhou, P. R. China
}

\subsection{INTRODUCTION}

Many applications of beams and pulses of light in modern optical technology, such as in long-distance communications, and laser micromachining, deep-field microscopy, plasma-channel generation, and laser writing of waveguides, gratings, and photonic crystals (to cite only a few), will benefit heavily from the use of particle-like waves. This term refers to waves that are capable of defeating diffraction spreading and dispersion broadening induced by the host material medium. while maintaining their spatiotemporal localization along sufficiently large, ideally infinite propagation distances. 
In the field of nonlinear optics, the achievement of localized and stationary propagation relies on the development of spatiotemporal solitary waves or solitons (also called "light bullets"), in which linear spreading (diffraction and material dispersion) is balanced by the compression supplied by the nonlinear response of the material. Although one-dimensional (temporal) solitary propagation has been achieved and has found broad technological applications in fiber communications, extension to the three-dimensional case has proved elusive to date, due to intrinsic soliton instabilities.

In linear optics, instead, the road toward the light bullet has followed the course of source design and spatiotemporal wave conformation by optical elements. Interest in the linear approach grew considerably from the introduction, or revival [1], of the Bessel or axicon beam [2.3], a (weakly) localized Bessel-shaped interference pattern that propagates without any diffraction change and from its generation in practical settings (see. e.g., $[2,4-6]$ ). Ideally, the Bessel beam is generated by the superposition of monochromatic plane waves with wave vectors distributed over the surface of a cone. Many generalizations and modifications of Bessel beams have been introduced. as Bessel beams of higher order, vectorial Bessel beams, apodized (finite power) Bessel-Gauss beams [7], partially coherent conical beams [8], Hankel beams [9], and the intriguing property of self-reconstruction of Bessel beams beyond obstacles [ 10$]$ have found unexpected application as optical tweezers for microscopic particle trapping and manipulation [11].

$X$-waves, introduced first in acoustics [12,13] and later demonstrated in optics [14] with the name Bessel $\mathrm{X}$-waves, represent the generalization of Bessel beams to the nonmonochromatic case (see [15] for a review), and hence the first type of truly spatiotemporal localized and stationary wave packet. At the same time. focus wave modes, introduced by different means [16.17] as a new type of spatiotemporal wave localization, were later demonstrated to belong also to the polychromatic Bessel beam family [18] and were subsequently demonstrated in real setups [19].

Whereas for $\mathrm{X}$-waves all monochromatic Bessel beam constituents have the same cone angle, focus wave modes present inherently cone-angle dispersion, that is, dependence of cone angle with frequency. This difference is essential for further generalization of polychromatic Bessel beams for the achievement of localization and stationarity in a dispersive material. In free-space or dispersion-less materials, coneangle dispersion is not needed for stationarity but is essential in dispersive materials. Stationarity requires the introduction of a suitable amount of cone-angle dispersion that balances material dispersion. Localized and stationary propagation in dispersive media was first observed in $[20,21]$ with Bessel X-pulses endowed by a small amount of cone-angle dispersion. The same effect was described further to also be attainable with other cone-angle dispersion configurations, as that in pulsed Bessel beams [22,23], envelope X-waves [24], spatiotemporal Gauss-Laguerre waves in media with anomalous dispersion [25], and to be possible also for broadband subcycle pulses [26].

The scope of this chapter is to collect, characterize, and classify all types of localized optical wave packets that present stationary propagation in a dispersive transparent medium. Throughout this chapter these waves are referred to as wave modes [27]. They key tool for the classification is the angular or transversal dispersion curve. 
First, we consider narrowband wave modes. These are shown (Section 8.2) to belong to two broad categories: hyperbolic modes, with X-shaped dispersion curve and spatiotemporal structure, if material dispersion is normal, or elliptic modes, with an O-shaped dispersion curve and spatiotemporal form, if material dispersion is anomalous [28]. In Section 8.3 we show that each wave mode can adopt the approximate form of (1) a pulsed Bessel beam (PBB), (2) an envelope focus wave mode (eFWM). or (3) an envelope $\mathrm{X}(\mathrm{eX})$ wave in normally dispersive media [envelope $\mathrm{O}(\mathrm{eO})$ wave in anomalously dispersive media], according to whether the mode bandwidth makes phase mismatch (PM), group-velocity mismatch (GVM) (with respect to a monochromatic plane wave), or defeated group velocity dispersion (GVD), respectively, to be the dominant mode characteristic on propagation. This classification allows us to understand the spatiotemporal features of wave modes in terms of a few parameters (the characteristic PM. GVM, and GVD lengths), including modes with mixed pulsed Bessel, focus wave mode, and X-like (O-like) structure.

In Section 8.4 we also describe broadband wave modes, whose dispersion curves and spatiotemporal forms are much more complex. The simple, symmetric. $\mathrm{X}$-like, and $\mathrm{O}$-like dispersion curves are actually approximations in narrow spectral bands to strongly asymmetric dispersion curves. Dispersion curves in broad spectral regions present as ymmetric $\mathrm{X}$ or $\mathrm{O}$-like forms, "fishlike" structure (formed by a closed loop plus a tail), or a single tail.

Our description is always paraxial. Although conical waves are usually described as nonparaxial waves, the paraxial approach turns out to lead to simpler expressions in terms of parameters linked directly to the physically relevant properties of the mode and the medium. We also use this approach because of its wider use in nonlinear optics. where unexpectedly, wave modes are finding wide application for understanding nonlinear phenomena. such as spatiotemporal effects in second harmonic generation [29-31] and collapse and filamentation of light pulses in Kerr media [32-34]. Wave modes appear to act as a kind of attractor of the wave dynamics described by the nonlinear Schrödinger equation. and have in fact been seen to be generated spontaneously in a number of nonlinear optics experiments [35-37], as also seen from numerical simulations [34,38]. For completeness, in Section 8.6 we compare the various paraxial and nomparaxial approaches.

\subsection{LOCALIZED AND STATIONARITY WAVE MODES WITHIN THE SVEA}

We start by considering the propagation of a three-dimensional wave packet $E(x, y, z, t)=A(x, y, z, t) \exp \left(-i s_{0} t+i k_{0} z\right)$ of a certain optical carrier frequency $\omega_{0}$, subject to the effects of diffraction and dispersion of the material medium. Within the SVEA, and up to second order in dispersion. propagation of the wave packet is ruled by the equation

$$
\partial_{\bar{c}} A=\frac{i}{2 k_{0}} \Delta_{\perp} A-i \frac{k_{0}^{\prime j}}{2} \partial_{\mathrm{\tau}}^{2} A
$$


where $z$ is the propagation direction, $\tau=t-k_{0}^{\prime} z$ is the local time, $\Delta_{\perp} \equiv \partial_{x}^{2}+\partial_{y}^{2}$, and $\left.k_{0}^{(i)} \equiv \partial_{\omega}^{(i)} k(\omega)\right|_{\omega_{0}}$. with $k(\omega)$ the propagation constant in the medium. Equation (8.1) is valid for a narrow envelope spectrum $\hat{A}\left(x_{\perp}, z, \Omega\right)$ around $\Omega \equiv \omega-\omega_{0}=0$, that is, for bandwidths

$$
\Delta \Omega \ll \omega_{0}
$$

Wave modes are stationary and localized solutions of Eq. (8.1) in the wide sense that the intensity does not depend on $z$ in a reference frame moving at some velocity. These solutions must then be of the form

$$
A(x, y, \tau, z)=\Phi(x, y, \tau+\alpha z) \exp (-i \beta z)
$$

The SVEA condition that $\left|\partial_{z} A\right| \ll k_{0}|A|$ requires the free parameters $\alpha$ and $\beta$ to be small, in the sense that

$$
\begin{aligned}
& |\alpha| \ll k_{0}^{i}, \\
& |\beta| \ll k_{0},
\end{aligned}
$$

so that the group velocity $1 /\left(k_{0}^{\prime}-\alpha\right)$ of the wave mode differs slightly from that of a plane pulse of the same carrier frequency in the same material, $1 / k_{0}^{\prime}$, and so does the phase velocity.

Wave modes may also be introduced in the spatiotemporal frequency domain as localized waves for which the axial component $k_{z}$ of the wave vector of the various monochromatic and plane-wave constituents varies linearly with frequency. This definition in Fourier space is equivalent to the ansatz (8.3) in space and time: Writing Eq. (8.3) as

$$
\begin{aligned}
A(x, y, \tau, z)= & \frac{1}{(2 \pi)^{3}} \int_{-\approx}^{\infty} d \Omega d k_{x} d k_{y} \hat{\Phi}\left(k_{x}, k_{y}, \Omega\right) \exp \left[i\left(k_{x} x+k_{y} y\right)\right] \\
& \times \exp \left\{-i \Omega\left[t-\left(k_{0}^{\prime}-\alpha\right) z\right]\right\} \exp (-i \beta z)
\end{aligned}
$$

where $\hat{\Phi}$ is the spatiotemporal Fourier transform of $\Phi$, and because $E=A$ exp $\left(-i c_{0} t+k_{0} z\right)$, it follows that the axial component of the wave vector of any monochromatic and plane-wave constituent is given by the linear relation

$$
k_{;}(\Omega)=\left(k_{0}-\beta\right)+\left(k_{0}^{i}-\alpha\right) \Omega
$$

Equation (8.1) with the wave-mode ansatz (8.3) yields

$$
\Delta_{\perp} \Phi-k_{0} k_{0}^{\prime \prime} \partial_{\tau}^{2} \Phi+2 i k_{0} \alpha \partial_{\tau} \Phi+2 k_{0} \beta \Phi=0
$$

for the reduced envelope $\Phi$, or for its spatiotemporal spectrum $\hat{\Phi}$.

$$
\left[-k_{\perp}^{2}+K^{2}(\Omega)\right] \hat{\Phi}=0
$$


where $k_{\perp}=\left(k_{x}^{2}+k_{y}^{2}\right)^{1 / 2}$ and where

$$
K(\Omega)=\sqrt{2 k_{0}\left(\beta+\alpha \Omega+\frac{1}{2} k_{0}^{\prime \prime} \Omega^{2}\right)} .
$$

This relation. of utmost importance in this chapter, and will be referred to as the (transversal) dispersion relation or curve of the wave mode since it relates the modulus $K$ of the transversal component of the wave vector with the detuning $\Omega$ of each monochromatic wave component from the carrier frequency $\omega_{0}$. The general solution of Eq. (8.9) is given by

$$
\hat{\Phi}\left(k_{x}, k_{y}, \Omega\right)=(2 \pi)^{3} \hat{g}\left(k_{x}, k_{y}, \Omega\right) \delta\left[k_{\perp}^{2}-K^{2}(\Omega)\right],
$$

where $\hat{g}\left(k_{x}, k_{y}, \Omega\right)$ is a spectral amplitude and $\delta(\cdot)$ is the Diracs delta function.

A single localized wave mode is determined by choices of $\alpha, \beta$ and of a spectral amplitude $\hat{g}\left(k_{x}, k_{y}, \Omega\right)$ yielding a localized wave. Choices of the form $\hat{g}\left(k_{x}, k_{y}, \Omega\right)=$ $\hat{g}\left(k_{\perp}, \Omega\right)$ lead to cylindrical symmetric or conical wave modes. which are of particular relevance in practice. Conical wave modes in space and time can be calculated by inverse spatial Fourier transform of Eq. (8.11) from the expression

$$
\begin{aligned}
A(r, \tau, z) & =\Phi_{\alpha, \beta}(r, \tau+\alpha z) \exp (-i \beta z) \\
& =\frac{1}{2 \pi} \int_{K(\Omega) \text { real }} d \Omega \hat{f}(\Omega) J_{0}[K(\Omega) r] \exp [-i \Omega(\tau+\alpha z)] \exp (-i \beta z),
\end{aligned}
$$

where the integral is limited to values of $\Omega$ such that $K^{2}(\Omega)$ is positive [i.e., $K(\Omega)$ is real, as indicated], $J_{0}(\cdot)$ is the Bessel function of the first kind and zero order; $r=\left(x^{2}+y^{2}\right)^{1 / 2}$, and $\hat{f}(\Omega)=\hat{g}(K(\Omega), \Omega) / 2$. In Eq. (8.12), the cylindrical symmetric wave modes exhibit their conical nature. They appear as superpositions of monochromatic Bessel beams whose cone-angle frequency dependence $\theta \simeq K(\Omega) / k(\omega)+\Omega)$ is determined by the dispersion relation (8.10). As $\hat{g}$, the function $\hat{f}$ is in practice an arbitrary function of detuning $\Omega$ related to the on-axis temporal form of the wave mode.

\subsubsection{Dispersion Curves Within the SVEA}

Most of the relevant properties of a wave mode are determined by its dispersion relation $K(\Omega)$. As shown in Fig. 8.1, the form of the dispersion curve $K(\Omega)$ reflects the underlying hyperbolic or elliptic geometries of the wave equation $(8.1)$ in the respective cases of propagation in media with normal or anomalous dispersion.

For normal dispersion $\left(k_{0}^{\prime \prime}>0\right)$, the dispersion curve is hyperbolic, often referred to as X-like. $K(\Omega)$ is a single-branch vertical hyperbola if $\beta>\alpha^{2} / 2 k_{0}^{\prime \prime}$ (Fig. 8.1a), and a two-branch horizontal hyperbola if $\beta<\alpha^{2} / 2 k_{0}^{\prime \prime}$ (Fig. 8.1b).

For anomalous dispersion $\left(k_{0}^{\prime \prime}<0\right), K(\Omega)$ takes real values only if $\beta>\alpha^{2} / 2 k_{0}^{\prime \prime}$, in which case the dispersion curve is an ellipse (Fig. $8.1 \mathrm{c}$ ), or O-like, for short. It is 

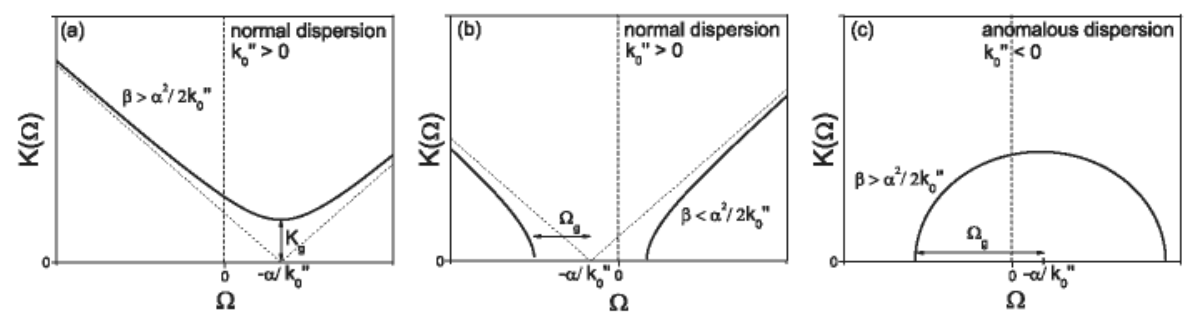

FIGURE 8.1 $\mathrm{X}$ - and $\mathrm{O}$-like dispersion curves of wave modes in a medium with (a) normal dispersion and $\beta>\alpha^{2} / 2 k_{0}^{\prime \prime}$; (b) normal dispersion and $\beta<\alpha^{2} / 2 k_{0}^{\prime \prime}$; (c) anomalous dispersion and $\beta>\alpha^{2} / 2 k_{0}^{\prime \prime}$ (exists only in this case).

also convenient to introduce the (real or imaginary) frequency and radial wave vector gaps

$$
\Omega_{g} \equiv \sqrt{\frac{\alpha^{2}}{k_{0}^{\prime \prime 2}}-\frac{2 \beta}{k_{0}^{\prime \prime}}}, \quad K_{g} \equiv \sqrt{-k_{0} k_{0}^{\prime \prime} \Omega_{g}^{2}} .
$$

When $\Omega_{g}$ and $K_{g}$ are real, they represent actual frequency and radial wave vector gaps in the dispersion curve $K(\Omega)$, as illustrated in Fig. 8.1. In any case, their moduli characterize the scales of variation of the frequency and radial wave vector in the dispersion curves.

\subsubsection{Impulse-Response Wave Modes}

Closely connected with the dispersion curves are the impulse response wave modes $\Phi_{\alpha, \beta}^{(i)}(r, \tau+\alpha z)$, or modes with $\hat{f}(\Omega)=1$. As seen in Fig. 8.2, the structure of $\Phi_{\alpha, \beta}^{(i)}$ in space and time closely resembles that of the dispersion curve in the $K-\Omega$ plane, but at radial and temporal scales of variation determined by the reciprocal quantities $\left|K_{g}\right|^{-1}$ and $\left|\Omega_{g}\right|^{-1}$, respectively. Equation (8.12) with $\hat{f}(\Omega)=1$ and the change $\Omega^{\prime}=\Omega+\alpha / k_{0}^{\prime \prime}$ yields

$$
\begin{aligned}
\Phi_{\alpha, \beta}^{(i)}(r, \tau+\alpha z)= & \frac{1}{2 \pi} \int_{K\left(\Omega^{\prime}\right) \text { real }} d \Omega^{\prime} J_{0}\left[K\left(\Omega^{\prime}\right) r\right] \exp \left[-i \Omega^{\prime}(\tau+\alpha z)\right] \\
& \times \exp \left[i \frac{\alpha}{k_{0}^{\prime \prime}}(\tau+\alpha z)\right]
\end{aligned}
$$

where

$$
K\left(\Omega^{\prime}\right)=\sqrt{k k_{0}^{\prime \prime}\left(\Omega^{\prime 2}+\frac{2 \beta}{k_{0}^{\prime \prime}}-\frac{\alpha^{2}}{k_{0}^{\prime \prime 2}}\right)} .
$$




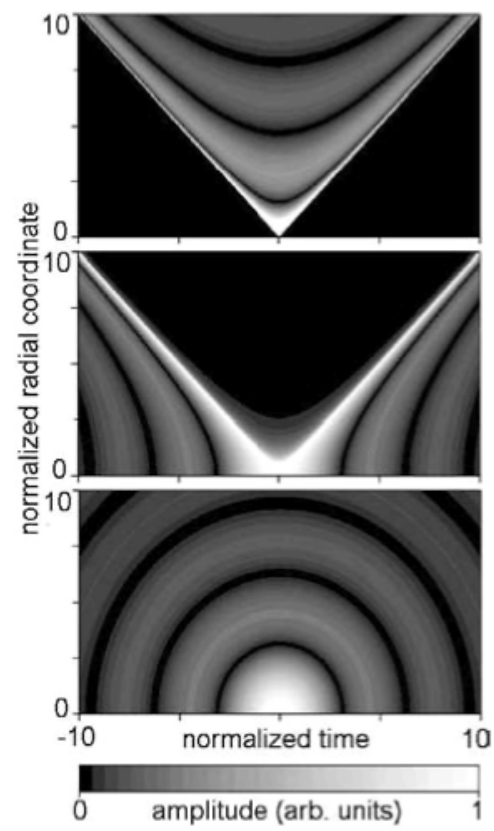

FIGURE 8.2 Contour plot for the amplitude $\left|\Phi_{\alpha, \beta}^{(i)}\right|$ of the impulse response wave modes. From top to bottom: normal dispersion $\left(k_{0}^{\prime \prime}>0\right)$ with $\beta>\alpha^{2} / 2 k_{0}^{\prime \prime}\left(\Omega_{g}\right.$ imaginary, transversal wave vector gap $K_{g}$ real); normal dispersion $\left(k_{0}^{\prime \prime}>0\right.$ ) with $\beta<\alpha^{2} / 2 k_{0}^{\prime \prime}$ (frequency gap $\Omega_{g}$ real, $K_{g}$ imaginary); anomalous dispersion ( $\left.k_{0}^{\prime \prime}<0\right)$ with $\beta>\alpha^{2} / 2 k_{0}^{\prime \prime}\left(\Omega_{g}\right.$ and $K_{g}$ real). Normalized local time and radial coordinate are defined as $\left|\Omega_{g}\right|(\tau+\alpha z)$ and $\left|K_{g}\right| r$, respectively.

The integral in Eq. (8.14) can be performed in all possible cases from formulas 6.677.3 (for $k_{0}^{\prime \prime}>0, \beta>\alpha^{2} / 2 k_{0}^{\prime \prime}$ ), 6.677.2 (for $k_{0}^{\prime \prime}>0, \beta<\alpha^{2} / 2 k_{0}^{\prime \prime}$ ), and 6.677.6 (for $k_{0}^{\prime \prime}<0$, $\beta>\alpha^{2} / 2 k_{0}^{\prime \prime}$ ) of [39], to yield the closed-form expression for impulse response modes:

$$
\begin{aligned}
& \Phi_{\alpha, \beta}^{(i)}(r, \tau+\alpha z) \\
& =\frac{1}{2 \pi}\left[\frac{\exp \left\{i\left[\sqrt{2 \beta / k_{0}^{\prime \prime}-\alpha^{2} / k_{0}^{\prime \prime 2}} \sqrt{k_{0} k_{0}^{\prime \prime} r^{2}-(\tau+\alpha z)^{2}}\right]\right\}}{\sqrt{k_{0} k_{0}^{\prime \prime} r^{2}-(\tau+\alpha z)^{2}}}+\text { c.c. }\right] \\
& \quad \times \exp \left[\frac{i \alpha}{k_{0}^{\prime \prime}}(\tau+\alpha z)\right],
\end{aligned}
$$

or in terms of the frequency and radial wave vector gap,

$$
\Phi_{\alpha, \beta}^{(i)}=\frac{1}{2 \pi}\left[\Omega_{g} \frac{\exp (i R)}{i R}+\text { c.c. }\right] \exp \left[\frac{i \alpha}{k_{0}^{\prime \prime}}(\tau+\alpha z)\right],
$$

where $R=\left[K_{g}^{2} r^{2}+\Omega_{g}^{2}(\tau+\alpha z)^{2}\right]^{1 / 2}$. 
As shown in Fig. 8.2 (top), for $k_{0}^{\prime \prime}>0$ and $\beta>\alpha^{2} / 2 k_{0}^{\prime \prime}$ ( $\Omega_{g}$ imaginary and $K_{8}$ real), the impulse response, $K$-gap, X-like wave mode is singular in the cone $r=$ $|(\tau+\alpha z)| / \sqrt{k_{i} k_{0}^{\prime \prime}}$, is zero for $r<|(\tau+\alpha z)| / \sqrt{k_{0} k_{0}^{\prime \prime}}$ (within the cone), and decays as $1 / r$ for $r>|(\tau+\alpha z)| / \sqrt{k_{0} k_{0}^{\prime \prime}}$ (out of the cone). The radial beatings in this region. of period $2 \pi / K_{g}$. are a consequence of the radial wave vector gap $K_{g}$.

Figure 8.2 (middle) shows the impulse response mode for $k_{0}^{\prime \prime}>0$ and $\beta<\alpha^{2} / 2 k_{0}^{\prime \prime}$ ( $\Omega_{g}$ real and $K_{g}$ imaginary). This $\Omega$-gap, X-like wave mode is, as in the previous case, singular at the cone $r=|(\tau+\alpha z)| / \sqrt{k_{0} k_{0}^{\prime \prime}}$, but damped oscillations are now temporal, of period $2 \pi / \Omega_{g}$, as corresponds to the frequency gap $\Omega_{g}$ in the dispersion curve. Out of the cone $\left[r>|(\tau+\alpha z)| / \sqrt{k_{0} k_{0}^{\prime \prime}}\right]$ the mode is exponentially localized.

Modes in media with anomalous dispersion [i.e., with $k_{6}^{\prime \prime}<0$ and $\beta>\alpha^{2} / 2 k_{0 \prime}^{\prime \prime}$ (real $\Omega_{8}$ and $K_{8}$ )] exhibit rather different characteristics [Fig. 8.2 (bottom)]. These modes are no longer singular and of $X$-type, but regular and, say. $O$-like. The damped oscillations decay temporally and radially as $1 / t$ and $1 / r$, respectively, with periods $2 \pi / \Omega_{g}$ and $2 \pi / K_{g}$. The absence of singularities is a consequence of the actual limitation that the elliptic dispersion curve imposes on the uniform spectrum $\hat{f}(\Omega)=1$.

\subsection{CLASSIFICATION OF WAVE MODES OF FINITE BANDWIDTH}

Numerical integration of Eq. (8.12) with a given dispersion curve (specified by the values of $\alpha, \beta$, and $k_{0}^{\prime \prime}$ ) but different (bell-shaped) spectral amplitude functions $\hat{f}(\Omega)$ also having different (but finite) bandwidths $\Delta \Omega$ [alternatively, numerical integration of

$$
\Phi_{\alpha, \beta}(r, \tau+\alpha z)=\int_{-\infty}^{\infty} d \sigma f(\sigma) \Phi_{\alpha, \beta}^{(i)}(r, \tau+\alpha z-\sigma),
$$

where $f(\tau)$ is the inverse Fourier transform of $\hat{f}(\Omega)$ ]. shows much richer and complex spatiotemporal features than is the case with infinite bandwidth. These features depend strongly on the choice of the spectral bandwidth $\Delta \Omega$, while no essentially new properties arise from the specific choice of $\hat{f}(\Omega)$ (Gaussian. Lorentzial, two-side exponential, etc.). Wave modes with finite bandwidth may exhibit mixed. more-or-less pronounced radial and temporal oscillations, along with incipient or strong X-wave (O-wave), focus wave mode or Bessel structure, as explained throughout this section (see also the figures that follow). The purpose of this section is to perform a simple comprehensive classification of wave modes in dispersive media. In the remainder of this chapter, $\Delta \Omega$ will refer to any suitable definition of the half-width of the bell-shaped spectral amplitude function $\hat{f}(\Omega)$.

Given a wave mode of parameters $\alpha$ and $\beta$ satisfying conditions (8.4) and (8.5), propagating in a dispersive material with GVD $k_{i, i}^{i i}$ and some spectral bandwidth $\Delta \Omega$ satisfying (8.2), we have found it convenient to define the following three characteristic lengths: 
1. The mode PM length

$$
L_{p} \equiv \frac{\mathbf{l}}{\beta}
$$

2. The mode walk-off, or GVM length,

$$
L_{\omega} \equiv \frac{1}{\alpha \Delta \Omega}
$$

measuring, respectively, the axial distances at which the mode becomes phase mismatched and walks off with respect to a plane pulse of the same spectrum in the same medium.

3. The GVD length

$$
L_{d} \equiv \frac{1}{k_{0}^{\prime \prime}(\Delta \Omega)^{2}}
$$

or distance at which the mode (invariable) duration differs significantly from that of the (broadening) plane pulse. Note that as defined, $L_{p}, L_{w}$, and $L_{d}$ can be positive or negative.

In terms of the mode lengths, the transversal dispersion relation $(8.10)$ takes the form

$$
K(\Omega)=\sqrt{2 k_{0}\left(L_{p}^{-1}+L_{u}^{-1} \Omega_{n}+\frac{1}{2} L_{d}^{-1} \Omega_{n}^{2}\right)} .
$$

where $\Omega_{n}=\Omega / \Delta \Omega$ is the normalized detuning, which ranges in $[-1,+1]$ for $\Omega$ within the bandwidth $\Delta \Omega$. Then they are the values of the mode lengths $L_{p}, L_{u}$, and $L_{d}$ that determine the form of the dispersion curve within the spectral bandwidth. and hence the parameters that determine the spatiotemporal structure of the mode, as shown throughout this section. We analyze here three extreme cases:

$$
\begin{array}{ll}
\left|L_{p}\right| \ll\left|L_{w}\right|,\left|L_{d}\right| & \text { PM-dominated case } \\
\left|L_{u}\right| \ll\left|L_{p}\right|,\left|L_{d}\right| & \text { GVM-dominated case } \\
\left|L_{d}\right| \ll\left|L_{p}\right|,\left|L_{w}\right| & \text { GVD-dominated case }
\end{array}
$$

that represent three well-defined, opposite experimental situations, and that also allow us to understand, at least qualitatively, the features of general, intermediate cases. The limiting cases in which $\left|L_{p}\right|,\left|L_{w}\right|$, and $\left|L_{d}\right|$ are infinitely small compared with the other two lengths correspond, respectively, to constant, parabolic, and linear (with normal dispersion) or elliptic (with anomalous dispersion) dispersion curves [see Fig. 8.3 (second row)], and lead to the prototype PBB, eFWM, and eX or eO wave modes, respectively [see Fig. 8.3 (first row)]. 

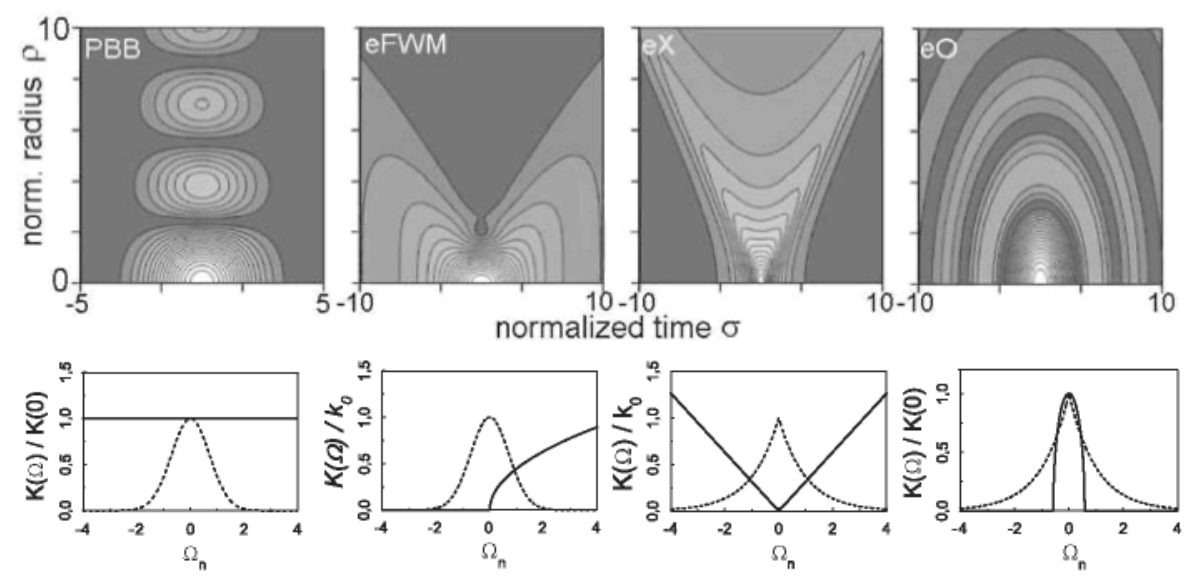

FIGURE 8.3 Top: Contour plots of the amplitude of the prototype PBB, eFWM, eX, and $\mathrm{eO}$ wave modes. Bottom: Corresponding constant, parabolic, linear, and elliptical dispersion curves (solid curves) within the spectra (dashed curves). Normalized coordinates are $\sigma=$ $(\tau+\alpha z) \Delta \Omega, \rho=r / r_{0}$, with $r_{0}=\left(2 k_{0} \beta\right)^{-1 / 2}$.

Generally speaking, modes of sufficiently long duration belong to, or participate mostly of, the PM-dominated (PBB-like) case, modes of some (still unspecified) intermediate duration belong to the GVM-dominated (eFWM-like) case, and extremely short modes to the GVD-dominated [eX-like (eO-like)] case, since $L_{p}$ is independent on bandwidth but $L_{p}$ and $L_{d}$ are inversely proportional to $\Delta \Omega$ and $\Delta \Omega^{2}$, respectively. Depending, however, on the relative values of $\alpha, \beta$, and $k_{0}^{\prime \prime}$ (particularly when one or two of them are very small), the GVM-dominated case, even the PM-dominated case, can extend down to the single-cycle regime, or on the contrary, the GVM-dominated case, even the GVD-dominated case, can apply to considerably long modes.

\subsubsection{Phase-Mismatch-Dominated Case: Pulsed Bessel Beam Modes}

Consider first modes with $\left|L_{p}\right| \ll\left|L_{w}\right|,\left|L_{d}\right|$. When $L_{p}>0$, the dispersion curve within the spectral bandwidth can be approached by the real constant value $K(\Omega) \simeq$ $\left(2 k_{0} L_{p}^{-1}\right)^{1 / 2}$, or

$$
K(\Omega) \simeq \sqrt{2 k_{0} \beta} \quad \text { if } \beta>0,
$$

regardless of whether the exact dispersion curve is an actual hyperbola or ellipse (as in Fig. 8.4), that is, independent of the sign of material group velocity dispersion. Wave modes under these conditions can have only superluminal phase velocity $(\beta>0)$ but can have super- or subluminal group velocity ( $\alpha>0$ or $\alpha<0$, respectively), and will adopt, from Eqs. (8.12) and (8.23), the approximate factorized form

$$
\Phi_{\alpha, \beta}(r, \tau+\alpha z) \simeq f(\tau+\alpha z) J_{0}\left(\sqrt{2 k_{0} \beta} r\right)
$$

of a PBB of transversal size on the order of $\left(2 k_{0} \beta\right)^{-1 / 2}$. 

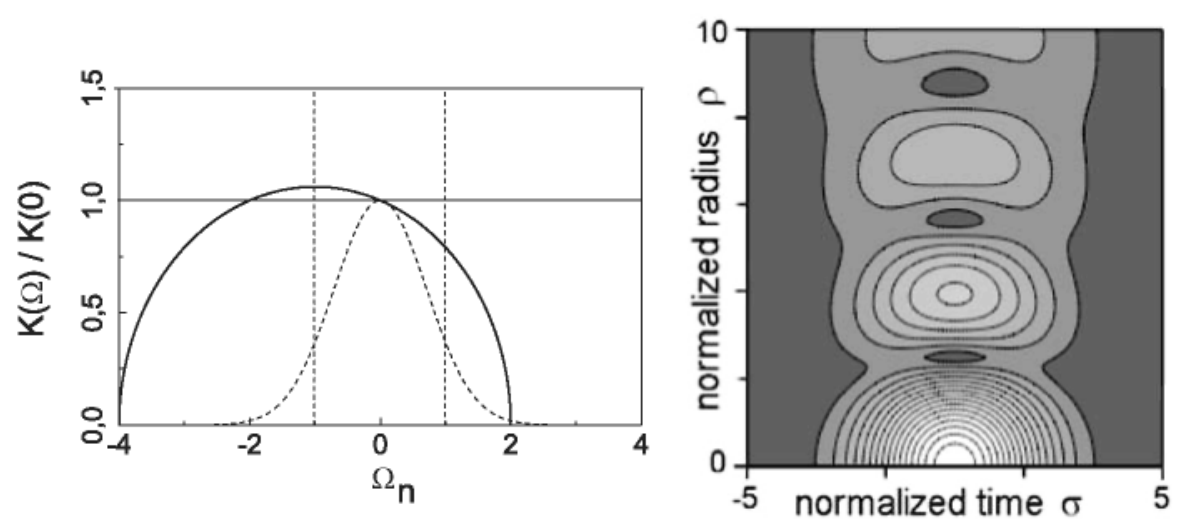

FIGURE 8.4 Left: Limiting dispersion curve for $\left|L_{p}\right| /\left|L_{w}\right| \rightarrow 0,\left|L_{p}\right| /\left|L_{d}\right| \rightarrow 0$ (thin line), for $L_{p} / L_{w}=-0.25, L_{p} / L_{d}=-0.25$ (thick curve), and Gaussian spectrum $\hat{f}(\Omega)=$ $\exp \left[-(\Omega / \Delta \Omega)^{2}\right]$ (i.e., $f(\tau) \propto \exp \left[-(2 \Delta \Omega \tau)^{2}\right]$ ) (dashed curve) for calculation of the wave modes. Right: Contour plot of the amplitude $\left|\Phi_{\alpha, \beta}\right|$ of the wave mode with the Gaussian spectrum and with $L_{p} / L_{w}=-0.25, L_{p} / L_{d}=-0.25$, calculated numerically from Eq. (8.12). Normalized coordinates are $\sigma=(\tau+\alpha z) \Delta \Omega, \rho=r / r_{0}$, with $r_{0}=\left(2 k_{0} \beta\right)^{-1 / 2}$.

Figure 8.3 shows the prototype PBB of this kind of wave mode [Eq. (8.24)] with a Gaussian spectrum $\hat{f}(\Omega)$, that is, the limiting case $\left|L_{p} / L_{w}\right|=0,\left|L_{p} / L_{d}\right|=0$. In Fig. 8.4 we also show, for comparison, the wave mode with $\left|L_{p} / L_{w}\right|=0.25$, $\left|L_{p} / L_{d}\right|=0.25$, and with the same Gaussian spectrum, obtained numerically from Eq. (8.12). We see that the wave mode preserves a spatiotemporal structure similar to that of the prototype PBB of Fig. 8.3, even if $\left|L_{p}\right|$ is not much smaller, but simply smaller than $\left|L_{w}\right|$ and $\left|L_{d}\right|$. Small differences can be understood as incipient focus wave mode and $\mathrm{O}$-wave-type behavior, as described in the following sections.

\subsubsection{Group-Velocity-Mismatch-Dominated Case: Envelope Focus Wave Modes}

When $\left|L_{w}\right| \ll\left|L_{p}\right|,\left|L_{d}\right|$, the dispersion curve within the bandwidth can be approached by the horizontal parabola $K(\Omega) \simeq\left(2 k_{0} L_{w}^{-1} \Omega_{n}\right)^{1 / 2}$ with vertex at $\Omega=0$, or

$$
K(\Omega) \simeq \sqrt{2 k_{0} \alpha \Omega}
$$

regardless of whether material dispersion is normal (as in Fig. 8.5) or anomalous. For modes with superluminal group velocity $(\alpha>0)$, the horizontal parabola is right-handed (as in Fig. 8.5) and is left-handed for subluminal modes $(\alpha<0)$. In any case, their spatiotemporal form can be approached by Eq. (8.12), with $K(\Omega)$ given by Eq. (8.25). Moreover, with the two-sided exponential spectrum 

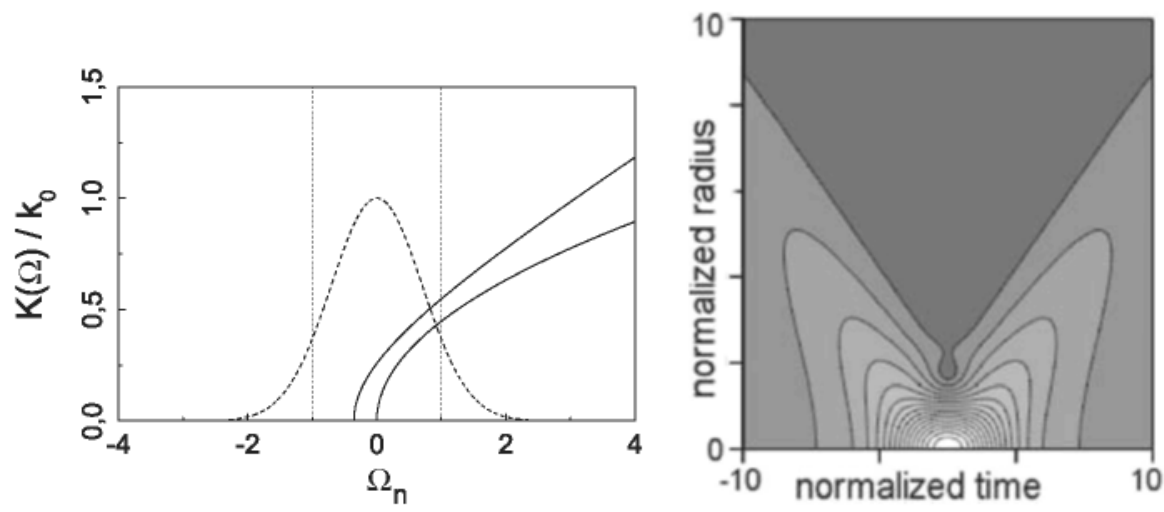

FIGURE 8.5 Left: Limiting dispersion curve for $L_{w}=10 / k_{0}, L_{w} / L_{p} \rightarrow 0, L_{w} / L_{d} \rightarrow 0$ (thin curve), for $L_{w}=10 k_{0}, L_{w} / L_{p}=1 / 3, L_{w} / L_{d}=1 / 3$ (thick curve), and Gaussian spectrum $\hat{f}(\Omega)=\exp \left[-(\Omega / \Delta \Omega)^{2}\right]$ (dashed curve) for calculation of the wave modes. Right: Contour plots of the amplitude $\left|\Phi_{\alpha, \beta}\right|$ of the wave mode with the Gaussian spectrum and with $L_{w} / L_{p}=1 / 3, L_{w} / L_{d}=1 / 3$, calculated numerically from Eq. (8.12). Normalized coordinates are $\sigma=(\tau+\alpha z) \Delta \Omega, \rho=r / r_{0}$, with $r_{0}=\left(2 / k_{0} \Delta \Omega|\alpha|\right)^{1 / 2}$.

$\hat{f}(\Omega)=(2 \pi / \Delta \Omega) \exp (-|\Omega| / \Delta \Omega)$, Eq. (8.12) yields

$$
\Phi_{\alpha, \beta}(r, \tau+\alpha z) \simeq \frac{-i \tau_{0}}{\tau+\alpha z-i \tau_{0}} \exp \left[\frac{i k_{0}|\alpha| r^{2}}{2\left(\tau+\alpha z-i \tau_{0}\right)}\right]
$$

for superluminal modes $(\alpha>0)$, and the complex conjugate of the right-hand side of Eq. (8.26) for subluminal modes $(\alpha<0)$. In Eq. (8.26), $\tau_{0} \equiv(\Delta \Omega)^{-1}$ characterizes the mode duration. The mode spot size at pulse center $(\tau+\alpha z=0)$ can be characterized by $r_{0}=\left(2 / k_{0} \Delta \Omega|\alpha|\right)^{1 / 2}$.

The functional form of the reduced envelope in Eq. (8.26) is similar to the fundamental Brittigham-Ziolkowski focus wave mode (FWM) [16,17], and as such will be called the envelope focus wave mode (eFWM). There are, however, important physical differences between them, which can be understood for the respective expressions of the complete fields $E$ of both types of waves:

$$
\begin{aligned}
E_{\alpha, \beta}(r, z, t) \simeq & \frac{-i \tau_{0}}{\tau+\alpha z-i \tau_{0}} \exp \left[\frac{i k_{0}|\alpha| r^{2}}{2\left(\tau+\alpha z-i \tau_{0}\right)}\right] \exp (-i \beta z) \\
& \times \exp \left(-i \omega_{0} t+i k_{0} z\right)
\end{aligned}
$$

for the envelope focus wave mode, and

$$
E(r, z, t)=\frac{-i \tau_{0}}{\tau-i \tau_{0}} \exp \left[\frac{i k_{0} r^{2}}{2 c\left(\tau-i \tau_{0}\right)}\right] \exp \left(-i \omega_{0} t-i k_{0} z\right),
$$

with $k_{0}=\omega_{0} / c$, for the fundamental FWM [17]. The fundamental FWM is a localized, stationary free-space wave whose envelope propagates at luminal group velocity $c$, 
whereas the carrier oscillations back-propagate at the same velocity $c$. The eFWM is also a stationary localized wave with the same intensity distribution as that of the fundamental FWM. but propagates in a dispersive medium with super- or subluminal group velocity $1 /\left(k_{0}^{\prime}-\alpha\right)$, and carrier oscillations propagate in the same direction.

Figure 8.3 shows the prototype eFWM of this type of wave modes, obtained from numerical integration of $\mathrm{Eq}$. (8.12) with the approximate dispersion curve $K(\Omega)=\sqrt{2 k_{0} \alpha \Omega}$ (i.e.. in the limiting case $\left|L_{w} / L_{p}\right|=0,\left|L_{w} / L_{d}\right|=0$ ) and a Gaussian spectrum. To pursue the validity of the model eFWM to describe this type of wave mode, we have also evaluated the wave mode field in some nonlimiting cases with the same Gaussian spectrum. Even for the relatively large ratios $\left|L_{w} / L_{p}\right|=1 / 3$, $\left|L_{w} / L_{d}\right|=1 / 3$, in which case the dispersion curve departs significantly from the limiting one (the thick and thin curves in Fig. 8.5), the wave mode calculated (Fig. 8.5) is nearly indistinguishable from the prototype eFWM (Fig. 8.3), exhibiting a similar eFWM structure along with some incipient $\mathrm{eX}$-wave behavior because of the actual hyperbolic form (not parabolic) of the dispersion curve, as explained in the next section.

\subsubsection{Group-Velocity-Dispersion-Dominated Case: Envelope X-and Envelope O-Modes}

Normal Group-Velocity Dispersion: Envelope $X$-Waves We consider finally modes with $\left|L_{d}\right| \ll\left|L_{p}\right|,\left|L_{w}\right|$, or modes of sufficiently short duration, or propagating in a medium with large enough GVD. When material dispersion is normal $\left(k_{0}^{i \prime}>0\right)$, the dispersion curve within the bandwidth approaches the X-shaped curve

$$
K(\Omega) \simeq \sqrt{k_{0} k_{0}^{\prime \prime}}|\Omega|
$$

of the limiting case $\left|L_{d} / L_{p}\right|,\left|L_{t l} / L_{w^{\prime}}\right|=0$ [Fig. 8.6(thin line)]. The actual dispersion curve of a mode may be shifted slightly toward negative frequencies (as in Fig. 8.6) or positive frequencies for modes with superluminal $(\alpha>0)$ or subluminal $(\alpha<0)$ group velocity, respectively. For modes with $\beta>0, K(\Omega)$ is real everywhere (Fig. 8.6), but for modes with $\beta<0$ there is a narrow frequency gap about $\Omega=0$. A prototype wave mode for this case can be obtained by introducing the approximate dispersion curve of $\mathrm{Eq}$. (8.29) into Eq. (8.12). With the two-sided exponential spectrum $\hat{f}(\Omega)=(2 \pi / \Delta \Omega) \exp (-|\Omega| / \Delta \Omega)$, we obtain

$$
\Phi_{\alpha . \beta}(r, \tau+\alpha z) \simeq \operatorname{Re}\left\{\frac{\tau_{0}}{\sqrt{k_{0} k_{0}^{\prime \prime} r^{2}+\left[\tau_{0}+i(\tau+\alpha z)\right]^{2}}}\right\},
$$

where $\tau_{0} \equiv(\Delta \Omega)^{-1}$ measures the pulse duration. Equation (8.30) is the eX-wave described in [24] as an exact, stationary, and localized solution of the paraxial wave equation with luminal phase and group velocities $(\alpha=\beta=0)$ in media with normal GVD. The eX-wave (8.30) is understood here as an approximate expression for modes with $\alpha, \beta$ such that $\left|L_{d} / L_{p}\right| \ll 1,\left|L_{d} / L_{w}\right| \ll 1$. The spatiotemporal form of the eXwave is shown in Fig. 8.3. For $L_{t} / L_{p}=1 / 6(\beta>0), L_{t} / L_{u^{\prime}}=1 / 6$ (thick curve 

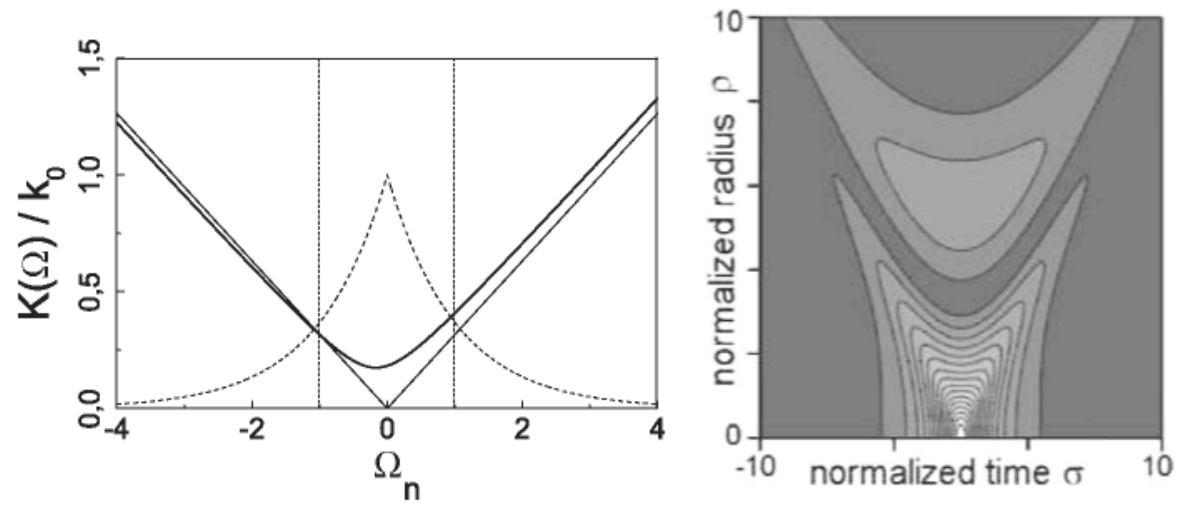

FIGURE 8.6 Left: Limiting dispersion curve for $L_{d}=10 / k_{0}, L_{d} / L_{p} \rightarrow 0, L_{d} / L_{w} \rightarrow 0$, with $L_{d}>0$ (thin line), for $L_{d}=10 / k_{0}, L_{d} / L_{p}=1 / 6, L_{d} / L_{w}=1 / 6$ (thick curve), and twosided exponential spectrum $\hat{f}(\Omega)=\exp (-|\Omega| / \Delta \Omega)$ (dashed curve). Right: Contour plot of the amplitude $\left|\Phi_{\alpha, \beta}\right|$ of the wave mode with the two-sided exponential spectrum and with $L_{d} / L_{p}=1 / 6, L_{d} / L_{w}=1 / 6$. Normalized coordinates are $\sigma=(\tau+\alpha z) \Delta \Omega, \rho=r / r_{0}$, with $r_{0}=\left(k_{0} k_{0}^{\prime \prime} \Delta \Omega^{2}\right)^{-1 / 2}$.

in Fig. 8.6), the mode retains an X-shaped structure (Fig. 8.6) despite the fact that the dispersion curve differs significantly from the limiting curve (the thin curve in Fig. 8.6). Incipient PBB behavior, or radial oscillations, originates from the nearly horizontal dispersion curve in the central part of the spectrum.

Anomalous Group-Velocity Dispersion: Envelope O-Waves When $\left|L_{d}\right| \ll$ $\left|L_{p}\right|,\left|L_{w}\right|$ but GVD is anomalous, the dispersion curve within the bandwidth can be approached by the ellipse centered approximately on $\Omega=0$ (Fig. 8.7, thick and thin curve), given by the expression

$$
K(\Omega) \simeq \sqrt{2 k_{0}\left(\beta-\left|k_{0}^{\prime \prime}\right| \Omega^{2} / 2\right)} .
$$

Note that the term with $\beta$, no matter how small, must be retained to reproduce the realvalued part of the dispersion curve. The group velocity of the mode can be slightly subluminal $(\alpha<0)$ or superluminal $(\alpha>0)$, as in Fig. 8.7, but the phase velocity of these modes is always superluminal $(\beta>0)$. An approximate analytical expression for this type of mode can be obtained by introducing the approximate dispersion curve of Eq. (8.31) into Eq. (8.12). Under the condition $\left|L_{d}\right| \ll\left|L_{p}\right|$, the frequency gap $\Omega_{g} \simeq \sqrt{2 \beta /\left|k_{0}^{\prime \prime}\right|}$ is much smaller than $\Delta \Omega$, so that the amplitude spectrum $\hat{f}(\Omega)$ can be assumed to take a constant value in the integration domain of integral in Eq. (8.12), which then yields the expression

$$
\Phi_{\alpha, \beta} \simeq \frac{1}{\sqrt{k_{0}\left|k_{0}^{\prime \prime}\right| r^{2}+(\tau+\alpha z)^{2}}} \sin \left[\sqrt{2 \beta /\left|k_{0}^{\prime \prime}\right|} \sqrt{k_{0}\left|k_{0}^{\prime \prime}\right| r^{2}+(\tau+\alpha z)^{2}}\right]
$$



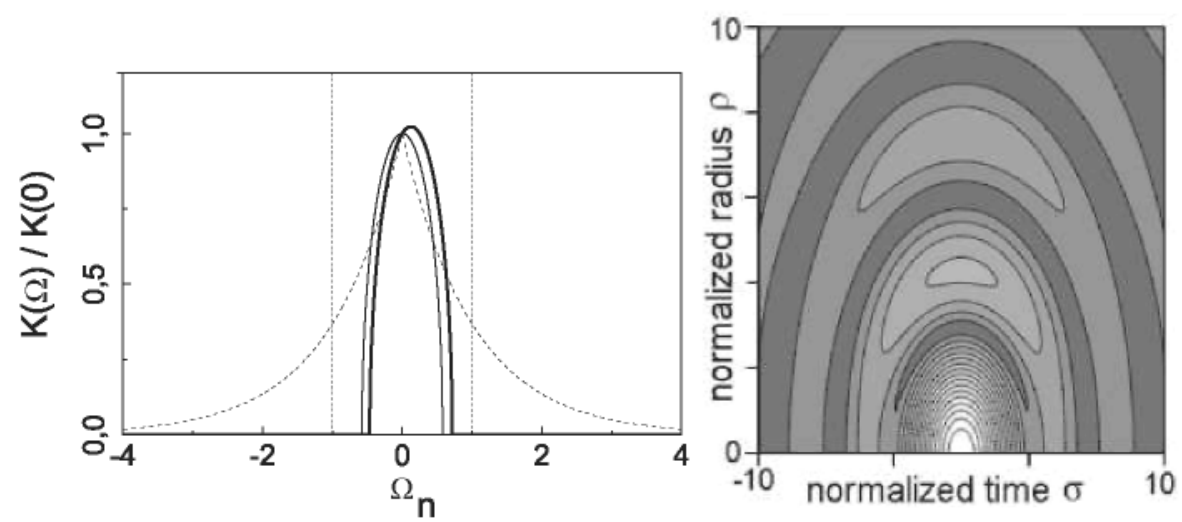

FIGURE 8.7 Left: Limiting dispersion curve for $\left|L_{d}\right| /\left|L_{p}\right| \rightarrow 0,\left|L_{d}\right| /\left|L_{w}\right| \rightarrow 0$, with $L_{d}<$ 0 (thin curve), for $L_{d} / L_{p}=-1 / 6, L_{d} / L_{w}=-1 / 8$ (thick curve), and the two-sided spectrum $\hat{f}(\Omega)=\exp (-|\Omega| / \Delta \Omega)$ (dashed curve). Right: Contour plot of the wave mode with $L_{d} / L_{p}=$ $-1 / 6, L_{d} / L_{w}=-1 / 8$ and the exponential spectrum, calculated numerically from Eq. (8.12). Normalized coordinates are $\sigma=(\tau+\alpha z) \sqrt{2 \beta /\left|k_{0}^{\prime \prime}\right|}, \rho=\sqrt{2 k_{0} \beta} r$.

which has the same form as the O-type impulse response mode in media with anomalous dispersion. Figure 8.3 shows its spatiotemporal form. For comparison, the wave mode with $L_{d} / L_{p}=-1 / 6, L_{d} / L_{w}=-1 / 8$ [Fig. 8.7 (thick curve)] and the twosided exponential spectrum [Fig. 8.7 (dashed curve)] was calculated from Eq. (8.12), and its O-shaped spatiotemporal form is also depicted in Fig. 8.7.

\subsection{WAVE MODES WITH ULTRABROAD BANDWIDTH}

Wave modes have been described above within the SVEA. In some circumstances (see below) wave modes with ultrabroad bandwidth, comparable to that of singlecycle pulses, have been observed, in which case the SVEA is no longer accurate. In this section we extend the description to a frame compatible with these broad spectra. Broadband wave modes show much richer spatiotemporal structures and dispersion curves than the simple $\mathrm{X}$ - and O-like narrowband wave modes, as shown in this section.

Our basic propagation equation is now

$$
\partial_{z} A=\frac{i}{2 k_{0}}\left(1+i \frac{k_{0}^{\prime}}{k_{0}} \partial_{\tau}\right)^{-1} \Delta_{\perp} A+i D A,
$$

derived in [40] within the slowly evolving wave approximation (SEWA) (but without performing the final approximation $k_{0}^{\prime} / k_{0} \simeq 1 / \omega_{0}$ [41]), which describes the propagation of pulses with duration $\Delta t$ (FWHM of $|A|^{2}$ ) as short as one carrier period $2 \pi / \omega_{0}$ under the effects of material dispersion up to the desired order, diffraction in 

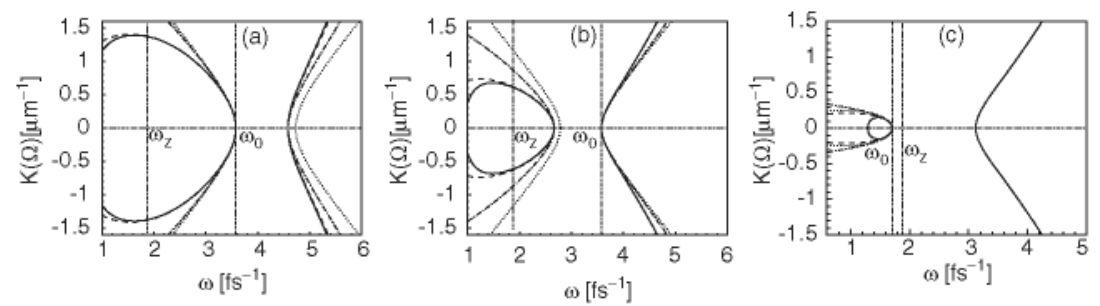

FIGURE 8.8 Dispersion curves of the wave modes in the normal [(a) and (b) $\lambda=0.527 \mu \mathrm{m}$ ] and anomalous [(c) $\lambda=1.7 \mu \mathrm{m}]$ dispersive region for water. (a) $\alpha=-0.032, \beta=0$; (b) $\alpha=$ $0.022, \beta=0$; (c) $\alpha=-0.001, \beta=0$.

the paraxial approximation, and spatiotemporal focusing (dependence of diffraction with frequency) up to first order. In Eq. (8.33),

$$
D=\sum_{m=2}^{\infty} \frac{k_{0}^{(m)}}{m !}\left(i \partial_{\tau}\right)^{m}
$$

is the dispersion operator.

Following a procedure similar to that described in Section 8.2 and using the wave mode ansatz (8.3) in Eq. (8.33), we obtain

$$
\Delta_{\perp} \Phi+2 k_{0}\left(1+i \frac{k_{0}^{\prime}}{k_{0}} \partial_{\tau}\right)\left[\beta+i \alpha \partial_{\tau}+i D\right] \Phi=0,
$$

from which the dispersion relation can be found to be

$$
K(\Omega)=\sqrt{2\left(k_{0}+k_{0}^{\prime} \Omega\right)[\beta+\alpha \Omega+\hat{D}(\Omega)]},
$$

where

$$
\hat{D}(\Omega)=\sum_{m=2}^{\infty} \frac{k_{0}^{(m)}}{m !} \Omega^{m}=k\left(\omega_{0}+\Omega\right)-k_{0}-k_{0}^{\prime} \Omega .
$$

Wave modes within the SEWA have the same expressions (8.11) and (8.12) as SVEA modes, but the dispersion relation is more accurate. Compared with the SVEA dispersion relation in Eq. (8.10), there is a new term in Eq. (8.36) standing for firstorder spatiotemporal focusing for broadband pulses. From the conditions of validity of the SEWA, the parameters $\alpha$ and $\beta$ should satisfy the same requirements as in Eqs. (8.4) and (8.5), whereas the condition (8.2) of narrow temporal spectrum is no longer required.

As an example, Fig. 8.8 shows wave-mode dispersion curves up to different degrees of approximation for water in normal dispersion [527-nm carrier wavelength, (a) and (b)] and anomalous dispersion [1100 $\mathrm{nm}$, (c)]. One of the interesting features of 
SEWA wave modes is that their dispersion curves (solid curves) can reproduce the structure of the broadband spatiotemporal spectra observed for light filaments [34,38], which suggests spontaneous formation of wave modes in the filamentation process. While dispersion curves within the SVEA (dotted curves) are always symmetric (X- or O-like) in the respective cases of normal and anomalous dispersion, SEWA dispersion curves are able to describe the strongly asymmetric structures observed. It is worth noting that the inclusion of third- or higher-order dispersion terms in SVEA wave modes (dash-dotted curves) renders the dispersion curves asymmetric but does not significantly improve the fitting to the observations. The inclusion of spatiotemporal focusing up to the first order is essential to obtain good agreement when dealing with such broad spectra. Generally, first-order spatiotemporal focusing and third-order dispersion suffice to reproduce the spectra observed when most of the spectrum lies on either the normal or anomalous spectral regions (dashed curves in Fig. 8.8a and b), but not when the spectrum covers both normal and anomalous regions (dashed curve in Fig. 8.8c, where the tail is missing), in which case all orders of dispersion are generally needed. In conclusion, the description of wave modes within the SEWA approximation appears to be the simplest, minimal extension of the SVEA description that is able to reproduce the observations in nonlinear experiments involving ultrabroad spectra.

\subsubsection{Classification of SEWA Dispersion Curves}

In this section we classify SEWA wave modes according to the possible forms of their dispersion curves. Whereas SVEA dispersion curves are always $\mathrm{X}$ - or $\mathrm{O}$-shaped, SEWA dispersion curves are much more complex. including distorted $X$ and $O$ shapes, "fishlike" and single-branch forms. For our classification, the only assumption on the medium is that second-order dispersion vanishes. as much, at only one frequency in the transparency spectral region of interest, and the only simplifying assumption is that $\beta \simeq 0$ [i.e. the dispersion curve passes through $(K=0, \Omega=0)$ ], which appears to correspond (as in Fig. 8.8) with the physically relevant situations.

The shape of the dispersion curve is seen to be determined primarily by its zeros. From $\mathrm{Eq}$. (8.36) with $\beta=0$, the zeros of $K(\Omega)$ are those of the function

$$
\hat{h}(\Omega)=\alpha \Omega+\hat{D}(\Omega)=k\left(\omega_{0}+\Omega\right)-k_{0}-\left(k_{0}^{\prime}-\alpha\right) \Omega
$$

(we do not consider the zero at $\Omega=-k_{0} / k_{0}^{i} \simeq-\omega_{0}$, i.e., at the de component $\omega=0$ ).

Distorted $X$-and O-Like Wave Modes Suppose first that GVD is normal (anomalous) for all frequencies of interest. This means that the concavity $\hat{h}^{\prime \prime}(\Omega)=k^{\prime \prime}\left(\omega_{0}+\right.$ $\Omega)$ ) of $\hat{h}(\Omega)$ is always positive (negative), that the slope $\hat{h}^{\prime}(\Omega)=k^{\prime}\left(\omega_{0}+\Omega\right)-\left(k_{0}^{\prime}-\right.$ $\alpha$ ) of $(\Omega)$ is a monotonous function, crossing consequently the $\Omega$-axis only once at a frequency close to the cartier (since $|\alpha|$ is small), and that $\hat{h}(\Omega)$ presents a single minimum (maximum) for normal (anomalous) dispersion at that frequency. Since Eq. (8.38) also requires that $\hat{h}(\Omega)$ cross the $\Omega$-axis at the carrier frequency $\Omega=0$, it 


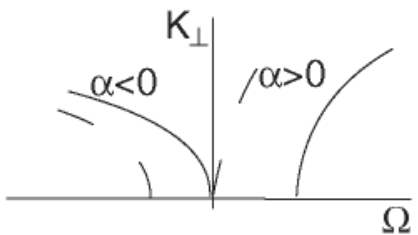

(a) normal dispersion

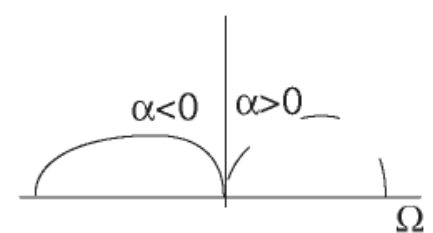

(b) anomalous dispersion

FIGURE 8.9 Typical dispersion curves of wave modes in a medium with only (a) normal or (b) anomalous dispersion.

follows that $\hat{h}(\Omega)$ must cross it once more, being positive outside (inside) the interval joining these two zeros for normal (anomalous) dispersion.

In conclusion, the dispersion curve $K$ for normal dispersion is real outside this interval, resembling a more or less distorted letter $\mathrm{X}$ with a frequency gap between the carrier and another frequency (Fig. 8.9a). For anomalous dispersion, $K$ is real between the carrier frequency and another frequency, displaying a distorted O-like form (Fig. 8.9b). The smaller the $|\alpha|$, the smaller the $\mathrm{X}$ gap and $\mathrm{O}$ width, reaching zero for $\alpha=0$. Distortion from X-and O-like SVEA wave modes is due to the effects of higher-order dispersion and the spatiotemporal focusing term, which always flattens the low-frequency part of the dispersion curve.

Fishlike and Single-Branch Wave Modes Suppose now the more general case that GVD changes from anomalous to normal at only one frequency $\omega_{z}$, and assume that $k_{0}^{\prime \prime \prime}\left(\omega_{z}\right)>0$, as in fused silica, water, and almost all optical materials of interests. This means that the $k^{\prime}(\omega)$ presents only one minimum at $\omega_{z}$ : in particular, for any carrier frequency $k_{0}^{\prime}-k^{\prime}\left(\omega_{z}\right)>0$.

In this case, the concavity of $\hat{h}(\Omega)\left(\hat{h}^{\prime \prime}(\Omega)=k^{\prime \prime}\left(\omega_{0}+\Omega\right)\right)$ changes from negative to positive only once at the flex point $\Omega_{z}=\omega_{z}-\omega_{0}$, where the slope takes its minimum (positive or negative) value $\hat{h}^{\prime}\left(\Omega_{z}\right)=k^{\prime}\left(\omega_{z}\right)-\left(k_{0}^{\prime}-\alpha\right)$. At the same time, from Eq. (8.38), $\hat{h}(\Omega)$ crosses the $\Omega$-axis at the carrier frequency with slope $\hat{h}^{\prime}(0)=\alpha$ (positive or negative). Thus, depending on whether $\alpha$ is smaller than zero, lies between 0 and $k_{0}^{\prime}-k^{\prime}\left(\omega_{z}\right)$, or is larger than $k_{0}^{\prime}-k^{\prime}\left(\omega_{z}\right)$, the dispersion curve may adopt the following forms.

Fishlike Wave Modes If $\alpha<0$ (subluminal wave modes), the slopes of $\hat{h}(\Omega)$ at the carrier $\omega_{0}$ and at the flex point $\omega_{z}$ are both negative. Thus, $\hat{h}(\Omega)$ must cross the $\Omega$-axis three times, and the dispersion curve $K(\Omega)$ adopts the fishlike structure illustrated in Fig. 8.10a and b (top), consisting of a closed loop and an isolated tail. This structure is the same regardless of whether the carrier frequency lies on the normal or anomalous spectral regions. However, the fishlike wave mode with carrier in anomalous dispersion behaves as a distorted O-like wave mode plus an added branch in the sense that the $\mathrm{O}$ narrows as $\alpha \rightarrow 0$ and the branch remains far from the O. In normal dispersion, the fishlike wave mode behaves as a distorted $\mathrm{X}$-like wave 

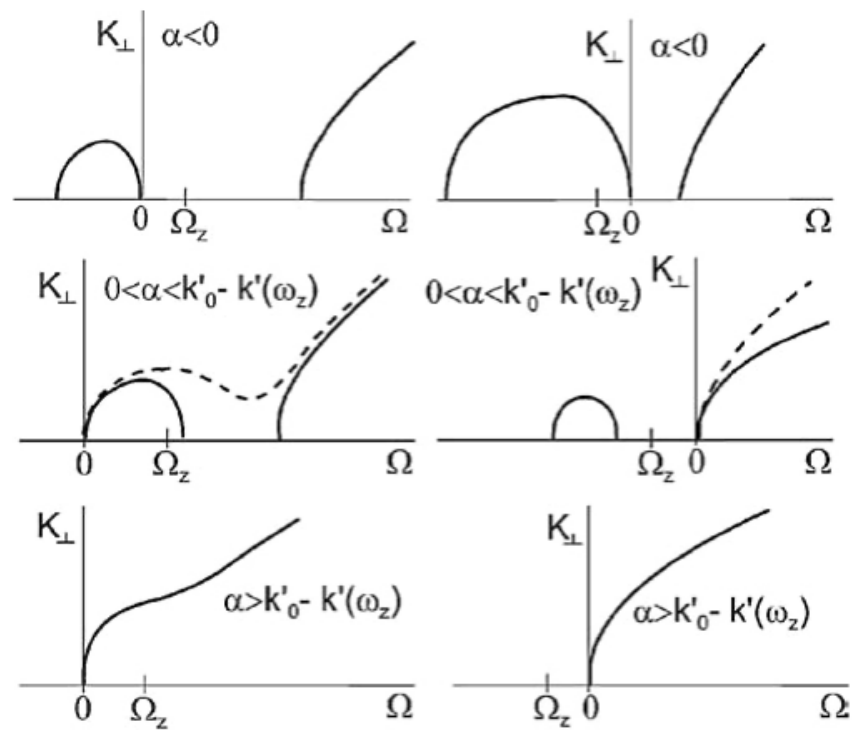

(a) anomalous dispersion

(b) normal dispersion

FIGURE 8.10 Typical dispersion curves of wave modes in a medium with one zero secondorder dispersion frequency.

mode featuring a closed branch, since the gap shortens for $\alpha \rightarrow 0$ and the tail closes far from the gap.

Transition Wave Modes If $0<\alpha<k_{0}^{\prime}-k^{\prime}\left(\omega_{z}\right)$ (slightly superluminal wave modes), the slope of $\hat{h}(\Omega)$ at the carrier $\omega_{0}$ is positive, but is negative at the flex point $\omega_{z}$. In this case, $\hat{h}(\Omega)$ will cross the $\Omega$-axis only once for the smaller values of $\alpha$, and three times for the larger values, the transition taking place at some $\alpha$ that can only be determined from knowledge of the material dispersion curve $k(\omega)$ at all frequencies. Accordingly, the dispersion curve for the smaller values of $\alpha$ will present a fishlike structure similar to that in the previous case, as shown in Fig. 8.10a and b (middle, solid curves). However, these dispersion curves differ substantially from the preceding case in that the $\mathrm{O}$ (in the anomalous region) and the gap (in the normal region) are placed in opposite sides of the carrier, and that the tail approaches the $O$ (anomalous) and the tail closing moves toward the gap (normal). For the larger values of $\alpha$, the $\mathrm{O}$ and tail join together (anomalous), and the tail closing reached the gap and disappeared (normal), the dispersion curve featuring only one branch, as shown in Fig. 8.10a and b (middle, dashed curves).

Tail-like Wave Modes If $\alpha>k_{0}^{\prime}-k^{\prime}\left(\omega_{z}\right)>0$ (superluminal wave modes), the slopes of $\hat{h}(\Omega)$ at the carrier and at the flex point are both positive, which means that $\hat{h}(\Omega)$ is a growing function everywhere that crosses the $\Omega$-axis only once at the carrier. The same applies to the dispersion curve $K$, which exhibits in anomalous 


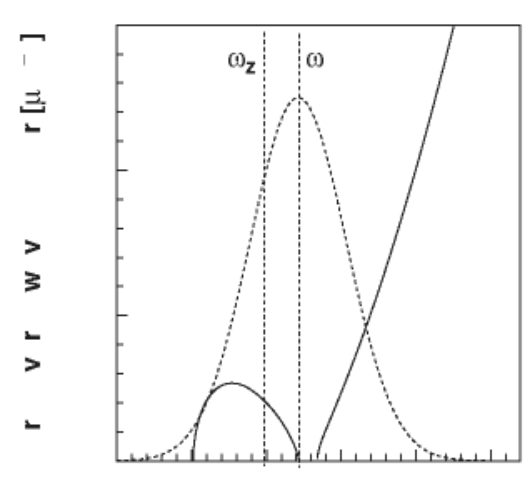

g $\quad$ fr q cy [f $\left.{ }^{-}\right]$

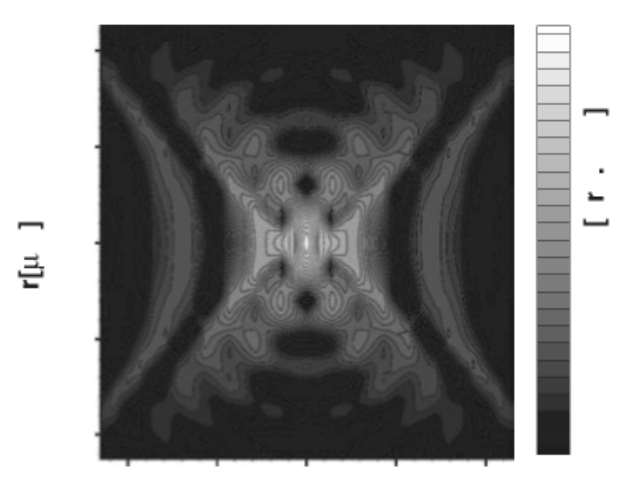

$\tau+\alpha \mathbf{z}[\mathbf{f}]$

FIGURE 8.11 Dispersion curve (a) and contour plot of the amplitude $|A|$ (b) of a single-cycle fishlike wave mode in the normal dispersion region of fused silica $\left(\omega_{0}=1.71 \mathrm{fs}^{-1}, \omega_{z}=1.48\right.$ $\left.\mathrm{fs}^{-1}, \alpha=-0.001 \mathrm{fs} / \mu \mathrm{m}, \beta=0\right)$. The thin-dotted curve is the spectrum of a single-cycle pulse $(\Delta t=3.67 \mathrm{fs})$.

and normal cases the form of a single monotonously increasing tail, as illustrated in Fig. 8.10a and b (bottom).

Further details can be known only from the specific form of the material dispersion. In the infrequent situation that $k^{\prime \prime \prime}\left(\omega_{z}\right)<0$ at the zero GVD frequency $\omega_{z}\left[k^{\prime}(\omega)\right.$ is maximum at $\omega_{z}$, and hence $k_{0}^{\prime}-k^{\prime}\left(\omega_{z}\right)<0$ ], all possible forms of the dispersion curves are identical but reflected about zero dispersion frequency $\Omega_{z}$, and fishlike wave modes are superluminal $(\alpha>0)$, transition wave modes are slightly subluminal [ $\left.k_{0}^{\prime}-k^{\prime}\left(\omega_{z}\right)<\alpha<0\right]$, and tail-like wave modes are subluminal $\left[\alpha<k_{0}^{\prime}-k^{\prime}\left(\omega_{z}\right)\right]$.

Finite bandwidth SEWA wave modes can be classified in a way similar to that in Section 8.3 . The only essentially different situation takes place when the bandwidth covers all three zeros of the dispersion curve. This may occur when the carrier frequency $\omega_{0}$ is close to $\omega_{z}$ and $\alpha$ is small. As an example, Fig. 8.11 shows the dispersion curve and spatiotemporal distribution of a single-cycle fishlike wave mode in a normal dispersion region of fused silica at carrier wavelength $1100 \mathrm{~nm}$ nearby the zero-GVD wavelength $1273 \mathrm{~nm}$. As seen, the spatiotemporal structure of the fishlike wave mode is a combination of an $\mathrm{X}$-wave and an $\mathrm{O}$-wave.

\subsection{ABOUT THE EFFECTIVE FREQUENCY, WAVE NUMBER, AND PHASE VELOCITY OF WAVE MODES}

One of the most striking features of wave modes is that their group velocity $1 /\left(k_{0}^{\prime}-\alpha\right)$ is independent of the spectrum $\hat{f}(\Omega)$. This property also holds for beating-like wave modes when discrete spectra are taken, even if composed of only two frequencies. However, for the phase velocity, this is no longer true. The frequency $\omega_{0}+\Omega$ of the actual carrier oscillations within the envelope of a wave mode is seen to depend on 
the spectrum $\hat{f}(\Omega)$, and so does the axial wave number $k_{z}(\Omega)=k_{0}-\beta+\left(k_{0}^{\prime}-\alpha\right) \Omega$ and the phase velocity $v_{p}=\left(\omega s_{0}+\Omega\right) / k_{z}(\Omega)$ along the propagation direction $z$. In this section we provide physically meaningful definitions of effective frequency, wave number, and phase velocity of a wave mode of spectrum $\hat{f}(\Omega)$.

We assume that $\hat{f}(\Omega)$ represents the spectrum of a bell-shaped pulse envelope $f(\tau)$. In the core of the wave mode (around the peak of the pulse), the effective frequency (shift from $\omega_{0}$ ) is often characterized by the "gravity center" of the wavemode spectrum: namely,

$$
\Omega_{\text {core }}=\frac{\int_{K(\Omega) \text { real }} d \Omega|\hat{f}(\Omega)| \Omega}{\int_{K(\Omega) \text { real }} d \Omega|\hat{f}(\Omega)|}
$$

which may differ from the central frequency of $\hat{f}(\Omega)$ because of the frequency gaps in the dispersion curve $K(\Omega)$. This yields an effective wave number

$$
k_{\text {core }}=k_{0}-\beta+\Omega_{\text {core }}\left(k_{0}^{\prime}-\alpha\right)
$$

and a phase velocity $v_{p}=\left(\omega_{0}+\Omega_{\text {core }}\right) / k_{\text {core }}$. This phase velocity reduces to $\omega_{0} /\left(k_{0}-\right.$ $\beta$ ) if the effective core frequency is $\Omega_{\text {core }}=0$ [e.g., if $\hat{f}(\Omega)$ is centered on $\Omega=0$ and $K(\Omega)$ does not have gaps].

If the dispersion curve has at least one zero with nonnegligible spectral amplitude $\hat{f}$. the frequency of the oscillations in the trailing and leading parts of the wave mode may differ significantly from that in the peak. In fact. the temporal behavior of the wave mode at large $|\tau|$ and on-axis $(r=0)$ is found from Eq. $(8.12)$ to be determined by the asymptotic expression

$$
\Phi(0, \tau+\alpha z) \sim-\frac{i}{\tau+\alpha z} \sum_{j=1}^{m} \hat{f}\left(\Omega_{j}\right) \exp \left[-i \Omega_{j}(\tau+\alpha z)\right]
$$

where $m$ is the number of zeros $\Omega$; of the dispersion curve with significant amplitude $\hat{f}\left(\Omega_{j}\right)$. The field amplitude decays as $1 /(\tau+\alpha z)$, while it oscillates at an effective frequency that can be determined from

$$
\Omega_{c l a d}=\frac{\sum_{j=1}^{n t}\left|\hat{f}\left(\Omega_{j}\right)\right| \Omega_{j}}{\sum_{j=1}^{m}\left|\hat{f}\left(\Omega_{j}\right)\right|}
$$

Then the effective wave number is given by

$$
k_{\text {clad }}=k_{0}-\beta+\Omega_{\text {clad }}\left(k_{0}^{\prime}-\alpha\right)
$$

and the phase velocity in the trailing and leading parts of the wave mode by $v_{p}=$ $\left(\omega_{0}+\Omega_{\text {clad }}\right) / k_{\text {clad }}$. 

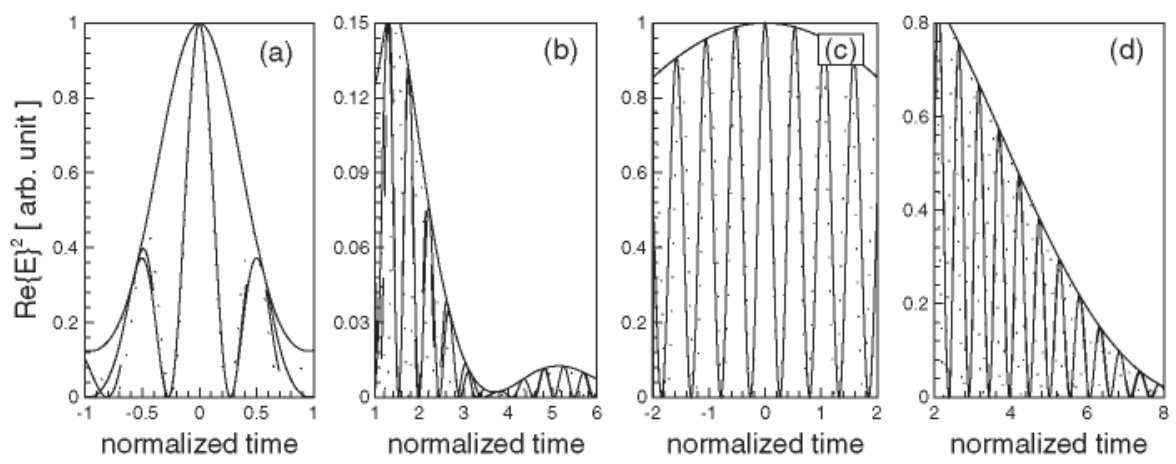

FIGURE 8.12 (a) and (b): On-axis real axial field Re $E$ (solid curves) of the wave mode in Fig. 8.8a. (c) and (d): The same for the wave mode in Fig. 8.8c. The normalized time is $(\tau+\alpha z) / \Delta t$.

If $\Omega_{\text {core }}=\Omega_{\text {clad }}$, the entire wave mode is characterized by a single effective frequency. This type of "good" wave mode can be constructed by an adequate choice of the spectral amplitude $\hat{f}(\Omega)$. For instance, good $\mathrm{X}$ or $\mathrm{O}$ wave modes require $\hat{f}$ to be centered on the X-gap or O-band.

In Fig. 8.12 we verify that these definitions provide physically meaningful values of wave-mode frequencies. The solid curves represent the time evolution of the onaxis field Re $E$ of the wave modes in Fig. 8.8a (X-like wave mode) and Fig. 8.12c (O-like wave mode). The dotted curves represent oscillations at $\omega_{0}$, and the dashed curves represent oscillations at $\Omega_{\text {core }}$ in parts (a) and (c) for the inner parts of these wave modes, and oscillations at $\Omega_{\text {clad }}$ in parts (b) and (d) for their outer parts. For the $\mathrm{X}$-like wave mode [(a) and (b)], the inner and outer carrier oscillations of the wave mode differ significantly, and are seen to be suitably characterized by $\Omega_{\text {core }}$ and $\Omega_{\text {clad }}$. The same can be said for the $\mathrm{O}$-like wave mode, although in this case both frequencies are very close.

\subsection{COMPARISON BETWEEN EXACT, SEWA, AND SVEA WAVE MODES}

A wave mode, independent of the approach used, is a localized wave in space and time in which the axial projection of its monochromatic plane-wave constituents presents a linear dependence with frequency:

$$
k_{z}(\Omega)=\left(k_{0}-\beta\right)+\left(k_{0}^{\prime}-\alpha\right) \Omega .
$$

In the cylindrically symmetric case, wave modes are often expressed as the superposition of Bessel beams:

$$
E(r, z, t)=\frac{1}{2 \pi} \int_{K \text { real }} d \omega \hat{f}\left(\omega-\omega_{0}\right) J_{0}[K(\omega) r] \exp \left(i k_{z}(\omega) z\right) \exp (-i \omega t)
$$


Introducing the linear relation (8.44) in Eq. (8.45), we obtain

$$
A(r, \tau, z)=\frac{1}{2 \pi} \int_{K(\Omega) \text { real }} d \Omega \hat{f}(\Omega) J_{0}[K(\Omega) r] \exp [-i \Omega(\tau+\alpha z)] \exp (-i \beta z)
$$

which is indeed identical for all approaches to wave modes [see Eq. (8.12)]. What differs in the various approaches to wave modes is the accuracy of the dispersion relation $K(\Omega)$, as required by the physical conditions.

In exact, nonparaxial wave modes, the transversal wave vector is determined simply by the constraint $k_{z}^{2}(\Omega)+K^{2}(\Omega)=k^{2}\left(\omega_{0}+\Omega\right)$, or

$$
\left(k_{0}-\beta\right)+\left(k_{0}^{i}-\alpha\right) \Omega=\sqrt{k^{2}\left(\omega_{0}+\Omega\right)-K^{2}},
$$

leading directly to

$$
K(\Omega)=\sqrt{k^{2}\left(\omega_{0}+\Omega\right)-\left[\left(k_{0}-\beta\right)+\left(k_{0}^{\prime}-\alpha\right) \Omega\right]^{2}},
$$

which is the exact nonparaxial dispersion relation.

In SEWA wave modes, instead, the paraxial approximation for each frequency is performed in Eq. (8.47):

$$
\left(k_{0}-\beta\right)+\left(k_{0}^{\prime}-\alpha\right) \Omega \simeq k\left(\omega_{0}+\Omega\right)-\frac{K^{2}}{2 k\left(\omega_{0}+\Omega\right)},
$$

and spatiotemporal coupling, or dependence of diffraction with frequency, is retained up to its first order by approaching $k\left(\omega_{0}+\Omega\right) \simeq k_{0}+k_{0} \Omega$ in the denominator of Eq. (8.49), that is.

$$
\left(k_{0}-\beta\right)+\left(k_{0}^{i}-\alpha\right) \Omega \simeq k\left(\omega_{0}+\Omega\right)-\frac{K^{2}}{2\left(k_{0}+k_{0}^{\prime} \Omega\right)},
$$

obtaining

$$
K(\Omega)=\sqrt{2\left(k_{0}+k_{0}^{i} \Omega\right)\left[k\left(\omega_{0}+\Omega\right)-\left(k_{0}-\beta\right)-\left(k_{0}^{i}-\alpha\right) \Omega\right]},
$$

which is the same as Eq. (8.36). The dispersion relation within the SVEA approximation results from further neglecting the spatiotemporal focusing term $k_{0}^{\prime} \Omega \simeq 0$ in Eq. (8.50). Up to second order in dispersion, we obtain the relation

$$
K(\Omega)=\sqrt{2 k_{0}\left(\beta+\alpha \Omega+\frac{1}{2} k_{0}^{\prime i} \Omega^{2}\right)},
$$

which is the same as Eq. $(8.10)$. 


\subsection{CONCLUSIONS}

In this chapter we have reviewed and classified wave modes, or the ultrashort polychromatic Bessel beams featuring localization and stationarity (diffraction- and dispersion-free) in a dispersive, transparent optical material, propagating with slightly super- or subluminal velocities. The key tool for the analysis is the transversal dispersion curve $K(\Omega)$ [or angular dispersion curve $\theta(\Omega) \simeq K(\Omega) / k_{0}$ ], which allows us also to understand the spatiotemporal wave-mode structure. The dispersion curves of SVEA wave modes are X-or O-like. Their spatiotemporal structure has been classified into three broad categories: PBB-like, eFWM-like, and eX-like (eO-like) modes, depending on the relative strength of their phase and group velocity mismatches with respect to a plane pulse, and their defeated GVD, as measured by the mode phasemismatch length $L_{p}$, group-mismatch length $L_{u t}$, and the dispersion length $L_{d}$. The dispersion curves of broadband wave modes, described from the SEWA approximation. are much more complex. In a medium with a zero-dispersion wavelength, they are fishlike or tail-like, in most cases reduced to distorted $X$ and $O$ wave modes if the bandwidth covers only the normal or anomalous dispersion regions. We believe that understanding the counterintuitive, particlelike properties of these fascinating waves is essential for the success of their technologic applications.

\section{REFERENCES}

1. J. A. Stratton, Electronagnetic Theory, McGraw-Hill, New York, 1941.

2. J. Durnin, J. J. Miceli, and J. H. Eberly, Phys. Rev. Lett. 58, 1499 (1987).

3. J. Durnin, J. Opt. Soc. Ant. A 4, 651 (1987).

4. J. Turunen, A. Vasara, and A. T. Friberg, Appl. Opt. 27, 3959 (1988).

5. A. Vasara, J. Turunen, and A. T. Friberg, J. Opt. Soc. Am. A 6, 1748 (1989).

6. L. Niggl, T. Lanzl, and M. Maier, J. Opt. SoC. Am. A 14, 27 (1997).

7. F. Gori, G. Guattari, and C. Padovani, Opt. Commmen. 64, 491 (1987).

8. A. V. Shchegrov and E. Wolf, Opt. Commin. 25, $141(2000)$.

9. S. Chávez-Cerda, G. S. McDonald, and G. H. C. New, Opt. Commun. 123, 225 (1996).

10. Z. Bouchal, J. Wagner, and M. Chlup, Opt. Commun. 151, 207 (1998).

11. V. Garcés-Chávez, D. McGloin, H. Melville, W. Sibbett, and K. Dholakia, Nature 419, 145 (2002).

12. J.-Y. Lu and J. F. Greenleaf, IEEE Trans. Ultrason. Ferroelectr. Freq. Control 37, 438 (1990).

13. J.-Y. Lu and J. F. Greenleat, IEEE Trans. Ultason. Ferroelectr Freq. Control 39, 19 (1992).

14. P. Saari and K. Reivelt, Phys. Rev. Lett. 79, 4135 (1997).

15. J. Salo, J. Fagerholm, A. T. Fribeg, and M. M. Solamaa, Phys. Rev: E 62, 4261 (2000).

16. J. N. Brittingham, J. Appl. Phys. 54, 1179 (1983).

17. R. W. Ziolkowski, Phy. Rev. A 39, 2005 (1989).

18. K. Reivelt and P. Saari, J. Opt. Soc. An. A 17, 1785 (2000). 
19. K. Reivelt and P. Saari, Phys. Rev, E 66, 05661 (2002).

20. H. Sonajalg and P. Saari, Opt. Lett. 21, 1162 (1996).

21. H. Sonajalg, M. Ratsep, and P. Saari, Opt. Lett. 22, 310 (1997).

22. M. A. Porras, Opt. Lett. 26, 1364 (2001).

23. M. A. Porras, R. Borghi, and M. Santarsiero, Opt. Conmunn. 206, 235 (2002).

24. M. A. Porras, S. Trillo, and C. Conti, Opt. Lett. 28, 1090 (2003).

25. S. Longhi, Phys. Rev. E 68, 066612 (2003).

26. S. Orlov, A. Piskarskas, and A. Stabinis, Opt. Lett. 27, 2167, 2103 (2002).

27. M. A. Porras and P. Di Trapani, Phws. Rev. E 69, 066606 (2004).

28. G. Valiulis, Optical Nonlinear Process Research Unit, internal report (unpublished).

29. P. Di Trapani, G. Valiulis, A. Piskarskas, O. Jedrkiewicz, J. Trull, C. Conti, and S. Trillo, Phys. Rev. Lett. 91, 093904 (2003); see also Phys. Rev. Focus, (Sept. 4, 2003), http://focus.aps.org/story/v12/st7.

30. O. Jedrkiewicz, J. Trull, G. Valiulis, A. Piskarskas, C. Conti, S. Trillo, and P. Di Trapani, Phys. Rev E 68, 026610 (2003).

31. G. Valiulis, J. Kilius, O. Jedrkiewicz, A. Bramati, S. Minardi, C. Conti, S. Trillo, A. Piskarskas, and P. Di Trapani, in OSA Trends in Optics and Photonics (TOPS), QELS 2001 Technical Digest, Vol. 57, Optical Society of America, Washington, DC, 2001.

32. M. A. Porras, A. Dubietis, E. Kucinskas, F. Bragher, V. Degiorgio, A. Couairon, D. Faccio, and P. Di Trapani, Opt. Lett. 30, 3398 (2005).

33. D. Faccio, F. Bragheri, and M. Cherchi, J. Opt. Soc. Ain. B 21, 296 (2004).

34. M. Kolesik, E. M. Wright, and J. V. Moloney, Phys. Rev. Lett. 92, 253901 (2004).

35. D. Faccioaaa, P. Di Trapani, S. Minardi, A. Bramati, F. Bragheri, C. Liberale, V. Degiorgio, A. Dubietis, and A. Matijosius, J. Opt. Soc. Am. B 22, 862 (2005).

36. D. Faccio, M. A. Porras, A. Dubietis, F. Bragheri, A. Couairon, and P. Di Trapani, Phys. Rev. Lett. 96, 193901 (2006).

37. M. A. Porras et al., Characterization of $\mathrm{O}$-shaped conical emission of light filaments in fused silica, J. Opt. Soc. Am. B 24, 581 (2007).

38. M. Kolesik, E. M. Wright, and J. V. Moloney, Opt. Express 13, 10729-10741 (2005).

39. I. S. Gradshteyn and I. M. Ryzhik, Table of Integrals, Series and Products, 4th ed., Academic Press, New York, 1965

40. T. Brabec and F. Krausz, Phys. Rev. Lett. 78, 3282 (1997).

41. M. A. Porras, Phys. Rev E 60, 5069 (1999). 